\title{
Procedures in the use of drones to validate the fire detection by satellites
}

\author{
Fabiano Morelli ${ }^{1}$, Heber Passos ${ }^{1}$, Joana Nogueira ${ }^{l}$, Marcelo Romão $^{l}$, Guilherme Martins ${ }^{l}$, \\ Alberto Setzer ${ }^{l}$, Wilfrid Schroeder ${ }^{2}$
}

\author{
${ }^{1}$ National Institute for Space Research (Inpe), São José dos Campos, Brasil \\ ${ }^{2}$ NOAA/NESDIS, USA \\ *e-mail: heber.passos@inpe.br
}

\begin{abstract}
Forest fires annually destroy extensive areas of vegetation, causing great environmental and economic damages. Many products derived from satellite observations have been used to monitor fire events. In Brazil, the Queimadas Program from INPE develops applications for the daily operational monitoring of hot spots detected by satellites. These detections are obtained with measurements from different sensors, which require specific algorithms and calibration parameters. The use of thermal sensor coupled to drone allows obtaining parameters with adequate spatial resolution. However, there are still no defined routines for obtaining these measures. The aim of this work is to propose a procedures protocol for the use of thermal sensor coupled to drone in experiments for validate the detection of hot spots by satellites. Based on our field experiences it is first necessary to have support from brigadiers, to instruct the teams with safety procedures to carry out the experiments. Secondly, i) to request authorization for the drone flights, in the SARPAS / DECEA system; ii) to check the weather conditions (cloud cover, wind speed and direction, etc.); iii) to determine the size of the burned area, which is inversely proportional to the spatial resolution of the satellite sensor studied; iv) to define the height of the drone's positioning, depending on the dimension of the chosen area and the sensor's field of view, and; v) to determine the sequence of the drone activation time, the start of the burning and the satellite imagery over the location. Finally, it is necessary to obtain quality thermal measures: i) to start the fire in advance for arrive to high temperatures during satellite imaging in the place of interest; ii) to consider the time to stabilize the drone and start acquiring the thermal data, before and after the peak of the satellite passage, and; iii) after the satellite has passed, to use the drone for measure the surrounding temperature (radius $=\sim 200 \mathrm{~m}$ ), obtaining a temperature reference in the surrounding areas. This protocol aims to standardize the experiments, improving the detection algorithms and providing improvements in the products presented in the Queimadas Program database.
\end{abstract}

Keywords: Thermal sensor; experimental burning; validation; fire; drone; calibration 